\title{
PID Controller Tuning Using Bode's Integrals *
}

\author{
Alireza Karimi, Daniel Garcia and Roland Longchamp ${ }^{\dagger}$
}

\begin{abstract}
A new method for PID controller tuning based on Bode's integrals is proposed. It is shown that the derivatives of amplitude and phase of a plant model with respect to frequency can be approximated by Bode's integrals without any model of the plant. This information can be used to design a PID controller for slope adjustment of the Nyquist diagram and improve the closed-loop performance. Besides, the derivatives can be also employed to estimate the gradient and the Hessian of a frequency criterion in an iterative PID controller tuning method. The frequency criterion is defined as the sum of squared errors between the desired and measured gain margin, phase margin and crossover frequency. The method benefits from specific feedback relay tests to determine the gain margin, the phase margin and the crossover frequency of the closed-loop system. Simulation examples and experimental results illustrate the effectiveness and the simplicity of the proposed method to design and tune the PID controllers.
\end{abstract}

Keywords: PID controller, relay feedback test, phase margin, gain margin, autotuning

\section{Introduction}

PID controllers are widely used in industrial plants and different methods for the design or the tuning of these controllers have been proposed in the literature $[8,16,3]$. The available methods are normally based on a first or a second-order model with time delay obtained from the time response or the measurement of multiple points on the frequency response of the process. The specifications are often expressed in terms of phase and/or gain margins $[7,12,6]$, because they are the classical measures of robustness and together with the crossover frequency, they represent the time domain performance of the closed-loop system as well. This problem, in general, even if the plant model is perfectly known, leads to a set of nonlinear equations for which no analytic solution is available. The traditional methods are the graphical algorithms using Bode plots which are not very suitable for autotuning of the PID controllers. More advanced methods try either to find a solution by numerical methods [12] or to simplify the equations using some approximations to

\footnotetext{
${ }^{*}$ This research work is financially supported by the Swiss National Science Foundation under grant No. 2100-064931.01

${ }^{\dagger}$ The authors are with the Laboratoire d'Automatique, Ecole Polytechnique Fédérale de Lausanne (EPFL), 1015 Lausanne, Switzerland. (email: alireza.karimi@epfl.ch)
} 
find an analytic solution [6]. The main drawback of these approaches is that the desired specifications are not necessarily achieved on the real system because of the approximations in modeling and/or equations. In addition, they do not lead to on-line tuning methods in order to meet the specifications for the real plant.

In 1984, Åström and Hägglund [7] proposed an automatic tuning method based on a simple relay feedback test which gives, using the describing function analysis, the critical gain and the critical frequency of the system. Other points on the Nyquist curve can be identified using a relay with hysteresis [7] or by introducing an adjustable time delay in the closed-loop system [10, 1]. A closed-loop relay test scheme was proposed in [15] which identifies directly the crossover frequency (the frequency at which the loop gain is equal to 1). After identifying a point on the frequency response of the plant, the so-called modified Ziegler-Nichols method can be used to move this point to another position in the complex plane [8]. Two equations for phase and amplitude assignment are obtained which can be solved to find the parameters of a PI or PD controller. For a PID controller, however, an additional equation should be introduced. In the modified Ziegler-Nichols method, the ratio between integral time $T_{i}$ and derivative time $T_{d}$ is chosen to be constant $\left(T_{i}=4 T_{d}\right)$ in order to have a unique solution. In a recent paper [9], it is shown that $T_{i}=2.5 T_{d}$ gives better results than the classical choice for a certain number of plant models. In [8], it was proposed finding the relation between $T_{i}$ and $T_{d}$ assuring a desired slope for the Nyquist curve of the loop transfer function at a given frequency. However, this relation depends inevitably on the derivatives of the plant transfer function with respect to frequency which are not known a priori.

The main contribution of this paper is in the use of Bode's integrals for PID controller tuning. The Bode's integrals [2] show the relation between the phase and the amplitude of minimum phase stable systems. It will be shown how these integrals can be used to approximate the derivatives of the amplitude and the phase of a system with respect to frequency at a given frequency. It is interesting to notice that the approximation is made only with the knowledge of the amplitude and the phase of the system at the given frequency and the system static gain. As a result, with a simple relay test, not only are the phase margin and the crossover frequency identified, but also the derivatives of the plant transfer function at crossover frequency are approximated with no parametric model of the plant. The derivatives can be used in the modified Ziegler-Nichols method to adjust the slope of the Nyquist curve at the crossover frequency. They can also be employed in an autotuning scheme for computing the gradient and the Hessian of a frequency criterion which is minimized iteratively. The frequency criterion can be defined as the sum of squared normalized errors between the desired and measured gain margin, phase margin and crossover frequency. An advantage compared with the autotuning methods based on a time criterion is that in each iteration a robustness margin is measured which can be used to make a stability test before applying the new controller to the system.

An iterative procedure for gain and phase margin adjustment for a PI controller was presented in [4]. The approach uses similar relay tests, but the gradient of the criterion is not calculated and an ad hoc 
algorithm is used, instead. As a result the algorithm converges much slower than the proposed method.

The paper is organized as follows: In Section 2 several relay feedback tests will be briefly reviewed. Section 3 shows how the Bode's integrals can be employed for slope adjustment in the modified Ziegler-Nichols method. An iterative tuning method for gain and phase margin adjustment together with simulation examples are presented in Section 4. An application of the proposed method to a three-tank system in Section 5 shows the effectiveness and fast convergence of the iterative method. Finally, Section 6 gives some concluding remarks.

\section{Relay feedback test}

The relay method is a well-known technique to identify useful points on the Nyquist curve by generating an appropriate oscillation with a relay feedback. In the standard relay method the controller is replaced by an on-off relay in closed-loop. For many systems there will be an oscillation when the control signal is a square wave and the process output is close to a sinusoid. The amplitude and the frequency of the limit cycle obtained give, respectively, the point where the Nyquist curve intersects the negative real axis and its corresponding frequency.

Relay feedback can also be applied to closed-loop systems including a linear controller. Fig. 1 shows an experiment where the output of the relay is the reference signal for the closed-loop system with an existing controller $K(s)$ and the output of the closed-loop system is fed back to the relay input. The identified

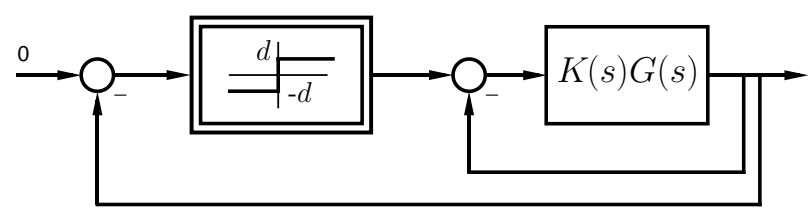

Figure 1: Relay experiment for gain margin measurement

point, obtained from the amplitude of the limit cycle, is on the intersection of the open-loop Nyquist curve with the segment $(-1,0)$. The gain margin is defined as the inverse of the amplitude of the identified point.

The experiment proposed in [15] and shown in Fig. 2 generates a limit cycle at the crossover frequency of the open-loop transfer function $K(j \omega) G(j \omega)$. The phase margin can be approximated by the amplitude and frequency of the generated limit cycle [11]. Let $\omega_{c}$ be the measured limit cycle frequency (equal to the crossover frequency), $a_{c}$ the relay input amplitude and $d$ the relay output amplitude. Then the phase of the identified point can be computed as follows:

$$
\angle K\left(j \omega_{c}\right) G\left(j \omega_{c}\right)=-2 \arctan \left(\frac{\pi a_{c} \omega_{c}}{4 d}\right)
$$




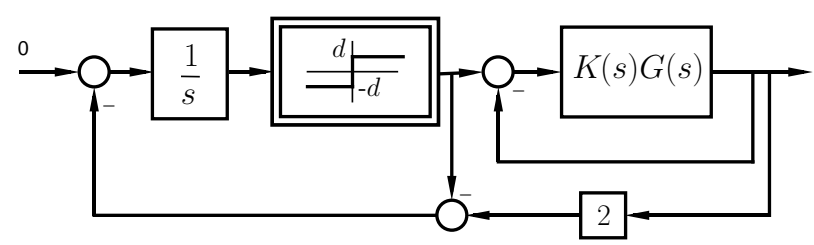

Figure 2: Relay experiment for phase margin measurement

The phase margin $\Psi$ is then given by

$$
\Psi=\pi-2 \arctan \left(\frac{\pi a_{c} \omega_{c}}{4 d}\right) .
$$

The proposed methods for measuring the gain and phase margins by the relay method have the drawback that the method of the describing function must be used for the identification of the frequencies and the points on the Nyquist curve of interest. This approach is approximative. Several methods are proposed to improve the precision of the approximations [16]. In this paper, an adaptive identification method to the critical gain proposed in [14] is used in all of the simulation examples and experiments. In this method the relay is replaced by a saturation nonlinearity and a time varying gain.

\section{Loop slope adjustment using Bode's integrals}

The slope of the Nyquist curve at the crossover frequency affects drastically the performance and robustness of the closed-loop system. In this section, first a formula is derived which gives the relation between $T_{i}$ and $T_{d}$ for obtaining the desired slope of the Nyquist curve of the loop transfer function at a given frequency. Then the Bode's integrals are used to approximate the derivatives of the plant transfer function. Finally, the parameters of a PID controller for the desired phase margin and slope at a given frequency are determined.

\subsection{Loop slope adjustment}

Consider the loop transfer function $L(j \omega)=G(j \omega) K(j \omega)$ where

$$
K(j \omega)=K_{p}\left(1+\frac{1}{j \omega T_{i}}+j \omega T_{d}\right)
$$

is the PID controller. The slope of the Nyquist curve of the loop transfer function $L(j \omega)$ at $\omega_{0}$ defined by $\psi$ is equal to the phase of the derivative of $L(j \omega)$ at $\omega_{0}$. The derivative of the loop transfer function with respect to $\omega$ is computed as follows:

$$
\frac{d L(j \omega)}{d \omega}=G(j \omega) \frac{d K(j \omega)}{d \omega}+K(j \omega) \frac{d G(j \omega)}{d \omega}
$$

Furthermore one has:

$$
\ln G(j \omega)=\ln |G(j \omega)|+j \angle G(j \omega)
$$


Differentiating this equation gives:

$$
\frac{d \ln G(j \omega)}{d \omega}=\frac{1}{G(j \omega)} \frac{d G(j \omega)}{d \omega}=\frac{d \ln |G(j \omega)|}{d \omega}+j \frac{d \angle G(j \omega)}{d \omega}
$$

On the other hand, the derivative of the controller with respect to $\omega$ is:

$$
\frac{d K(j \omega)}{d \omega}=j K_{p}\left(T_{d}+\frac{1}{\omega^{2} T_{i}}\right)
$$

Substituting Eqs (1), (4) and (5) into Eq. (2), one obtains:

$$
\frac{d L(j \omega)}{d \omega}=K_{p} G(j \omega)\left[j\left(T_{d}+\frac{1}{\omega^{2} T_{i}}\right)+\left(1+j\left(T_{d} \omega-\frac{1}{\omega T_{i}}\right)\right)\left(\frac{d \ln |G(j \omega)|}{d \omega}+j \frac{d \angle G(j \omega)}{d \omega}\right)\right]
$$

Hence, the slope of the Nyquist curve at $\omega_{0}$ is given by:

$$
\psi=\left.L \frac{d L(j \omega)}{d \omega}\right|_{\omega_{0}}=\varphi_{0}+\arctan \frac{\left(T_{d} T_{i} \omega_{0}^{2}+1\right)+\left(T_{d} T_{i} \omega_{0}^{2}-1\right) s_{a}\left(\omega_{0}\right)+s_{p}\left(\omega_{0}\right) T_{i} \omega_{0}}{s_{a}\left(\omega_{0}\right) T_{i} \omega_{0}-\left(T_{d} T_{i} \omega_{0}^{2}-1\right) s_{p}\left(\omega_{0}\right)}
$$

where $\varphi_{0}=\angle G\left(j \omega_{0}\right)$ and $s_{a}\left(\omega_{0}\right)$ and $s_{p}\left(\omega_{0}\right)$ are defined as follows:

$$
\begin{aligned}
& s_{a}\left(\omega_{0}\right)=\left.\omega_{0} \frac{d \ln |G(j \omega)|}{d \omega}\right|_{\omega_{0}} \\
& s_{p}\left(\omega_{0}\right)=\left.\omega_{0} \frac{d \angle G(j \omega)}{d \omega}\right|_{\omega_{0}}
\end{aligned}
$$

It is desired to adjust the slope of the Nyquist curve of the loop transfer function $L(j \omega)$ to a specified value $\psi$. Then straightforward calculation gives:

$$
T_{d}=\frac{s_{a}\left(\omega_{0}\right)-1+s_{p}\left(\omega_{0}\right) \tan \left(\psi-\varphi_{0}\right)-T_{i} \omega_{0}\left(s_{p}\left(\omega_{0}\right)-s_{a}\left(\omega_{0}\right) \tan \left(\psi-\varphi_{0}\right)\right)}{\omega_{0}^{2} T_{i}\left(1+s_{a}\left(\omega_{0}\right)+s_{p}\left(\omega_{0}\right) \tan \left(\psi-\varphi_{0}\right)\right)}
$$

In the next part, $s_{a}\left(\omega_{0}\right)$ and $s_{p}\left(\omega_{0}\right)$ are directly approximated using the Bode's integrals.

\subsection{Bode's integrals}

The relations between the phase and the amplitude of a stable minimum-phase system have been investigated for the first time by Bode [2]. The results are based on Cauchy's residue theorem and have been extensively used in network analysis. Two integrals are presented in this section. The first one, which is well known in the control engineering field, shows the relationship between the phase of the system at each frequency as a function of the derivative of its amplitude. But the second integral, to the best of the authors' knowledge, has been never used in the control design. The integral shows how the amplitude of the system at each frequency is related to the derivative of the phase and the static gain of the system.

\subsubsection{Derivative of amplitude}

Bode has shown in [2] that for a stable minimum-phase transfer function $G(j \omega)$, the phase of the system at $\omega_{0}$ is given by:

$$
\angle G\left(j \omega_{0}\right)=\frac{1}{\pi} \int_{-\infty}^{+\infty} \frac{d \ln |G(j \omega)|}{d \nu} \ln \operatorname{coth} \frac{|\nu|}{2} d \nu
$$


where $\nu=\ln \frac{\omega}{\omega_{0}}$. Since $\ln \operatorname{coth} \frac{|\nu|}{2}$ decreases rapidly as $\omega$ deviates from $\omega_{0}$, the integral depends mostly on $\frac{d \ln |G(j \omega)|}{d \nu}$ (the slope of the Bode plot) near $\omega_{0}$. Therefore, assuming that the slope of the Bode plot is almost constant in the neighborhood of $\omega_{0}, \angle G\left(j \omega_{0}\right)$ can be approximated by:

$$
\left.\angle G\left(j \omega_{0}\right) \approx \frac{1}{\pi} \frac{d \ln |G(j \omega)|}{d \nu}\right|_{\omega_{0}} \int_{-\infty}^{+\infty} \ln \operatorname{coth} \frac{|\nu|}{2} d \nu=\left.\frac{\pi}{2} \frac{d \ln |G(j \omega)|}{d \nu}\right|_{\omega_{0}}
$$

This property is often used in loop shaping where the slope of the amplitude Bode plot at the crossover frequency is limited to $-20 \mathrm{~dB} /$ decade in order to obtain approximately a phase margin of $90^{\circ}$. Here the measured phase of the system at $\omega_{0}$ is used to determine approximately the slope of the amplitude Bode $\operatorname{plot}\left(s_{a}\right)$ :

$$
s_{a}\left(\omega_{0}\right)=\left.\frac{d \ln |G(j \omega)|}{d \nu}\right|_{\omega_{0}}=\left.\omega_{0} \frac{d \ln |G(j \omega)|}{d \omega}\right|_{\omega_{0}} \approx \frac{2}{\pi} \angle G\left(j \omega_{0}\right)
$$

\subsubsection{Derivative of phase}

The second Bode's integral shows that the amplitude of a stable minimum-phase system can be determined uniquely from its phase and its static gain. More precisely, the logarithm of the system amplitude at $\omega_{0}$ is given by [2]:

$$
\ln \left|G\left(j \omega_{0}\right)\right|=\ln \left|K_{g}\right|-\frac{\omega_{0}}{\pi} \int_{-\infty}^{+\infty} \frac{d(\angle G(j \omega) / \omega)}{d \nu} \ln \operatorname{coth} \frac{|\nu|}{2} d \nu
$$

where $K_{g}$ is the static gain of the plant. In the same way, assuming that $\angle G(j \omega) / \omega$ is linear (in a logarithmic scale) in the neighborhood of $\omega_{0}$, one has:

$$
\begin{aligned}
\ln \left|G\left(j \omega_{0}\right)\right| & \approx \ln \left|K_{g}\right|-\left.\frac{\omega_{0}}{\pi} \frac{d(\angle G(j \omega) / \omega)}{d \nu}\right|_{\omega_{0}} \frac{\pi^{2}}{2} \\
\ln \left|G\left(j \omega_{0}\right)\right| & \approx \ln \left|K_{g}\right|-\frac{\pi \omega_{0}^{2}}{2}\left[\left.\frac{1}{\omega_{0}} \frac{d \angle G(j \omega)}{d \omega}\right|_{\omega_{0}}-\frac{\angle G\left(j \omega_{0}\right)}{\omega_{0}^{2}}\right]
\end{aligned}
$$

which gives:

$$
s_{p}\left(\omega_{0}\right)=\left.\omega_{0} \frac{d \angle G(j \omega)}{d \omega}\right|_{\omega_{0}} \approx \angle G\left(j \omega_{0}\right)+\frac{2}{\pi}\left[\ln \left|K_{g}\right|-\ln \left|G\left(j \omega_{0}\right)\right|\right]
$$

Note that for the systems containing an integrator, the static gain cannot be computed. For such systems, the static gain of the system without the integrator should be estimated and used in the above formula (note that the phase of the integrator is constant and its derivative is zero).

\subsubsection{Precision of the estimates}

The precision of the estimates of the derivatives of amplitude and phase depends on the system dynamics and on the frequency at which the experiments are performed. However, extensive simulations on the typical models of industrial plants have shown that the absolute normalized error of the estimates is in an acceptable range. In order to give an idea about the precision of the estimates, let us consider the following system:

$$
G(s)=\frac{1}{(s+1)^{n}}
$$


where $n$ is a positive integer. The true values of $s_{a}(\omega)$ and $s_{p}(\omega) / \omega$ computed on the basis of the model for different frequencies, are compared with the estimated ones based on the Bode's integrals in Fig. 3 and Fig. 4, respectively. It can be observed that the maximum of the absolute normalized error does not exceed 0.1 for this system.

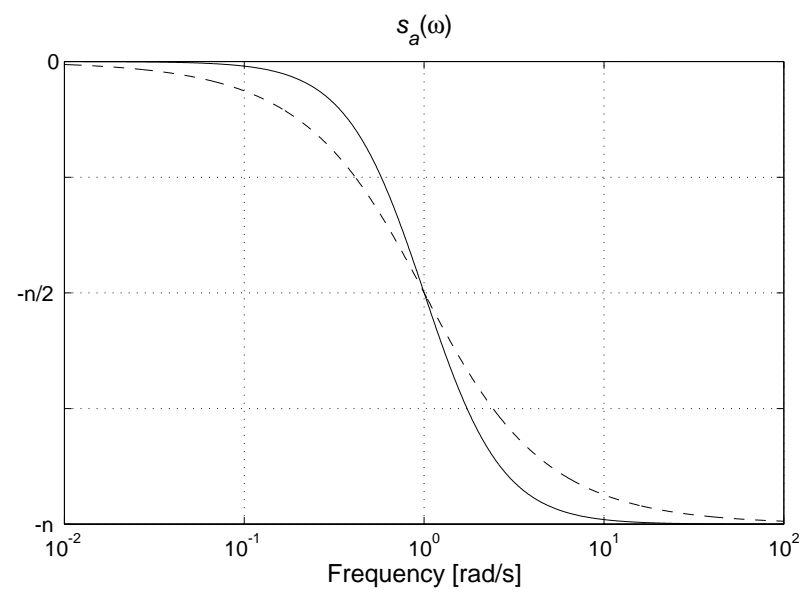

Figure 3: Comparison of true $s_{a}(\omega)$ and the estimated one based on Bode's integral (solid line: true values, dashed line: estimates )

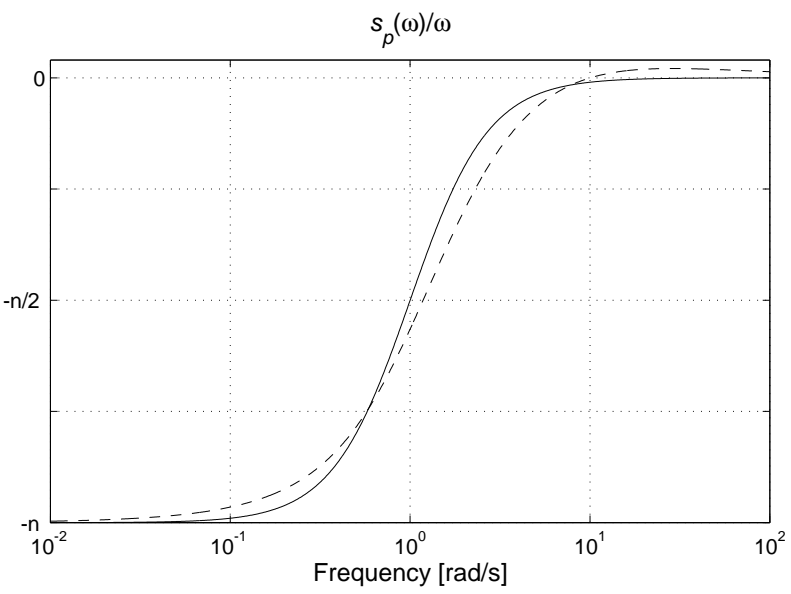

Figure 4: Comparison of true $s_{p}(\omega) / \omega$ and the estimated one based on Bode's integral (solid line: true values, dashed line: estimates )

Similar results can be obtained for the systems presented by several first-order models in cascade. For oscillatory systems, the estimation may be poor in certain frequencies, but in general the results remain satisfactory. For non-minimum-phase systems the Bode's integrals are no longer valid and the proposed formulas give incorrect results. However, it will be shown next that the pure time delay has no effect on the estimation of $s_{p}$ and its effect on the estimation of $s_{a}$ can be neglected if it is small with respect to 
the dominant time constant of the system.

\subsubsection{Effect of pure time delay}

Consider the following system with a pure time delay $\tau$ :

$$
G_{\tau}(j \omega)=G(j \omega) e^{-j \tau \omega}
$$

where $G(j \omega)$ is a stable minimum-phase system. Differentiating the amplitude and phase of $G_{\tau}(j \omega)$ with respect to $\omega$ gives:

$$
\frac{d \ln \left|G_{\tau}(j \omega)\right|}{d \omega}=\frac{d \ln |G(j \omega)|}{d \omega} \quad, \quad \frac{d \angle G_{\tau}(j \omega)}{d \omega}=\frac{d \angle G(j \omega)}{d \omega}-\tau
$$

Now using the Bode's integral from Eq. (12), the phase of $G_{\tau}(j \omega)$ at $\omega_{0}$ is approximated by:

$$
\angle G_{\tau}\left(j \omega_{0}\right)=\angle G\left(j \omega_{0}\right)-\left.\tau \omega_{0} \approx \frac{\pi}{2} \frac{d \ln |G(j \omega)|}{d \omega}\right|_{\omega_{0}}-\tau \omega_{0}
$$

Then $s_{a}\left(\omega_{0}\right)$ and $s_{p}\left(\omega_{0}\right)$ for a system including a pure time delay are computed as follows:

$$
\begin{aligned}
s_{a}\left(\omega_{0}\right) & =\left.\omega_{0} \frac{d \ln \left|G_{\tau}(j \omega)\right|}{d \omega}\right|_{\omega_{0}} \approx \frac{2}{\pi}\left(\angle G_{\tau}\left(j \omega_{0}\right)+\tau \omega_{0}\right) \\
s_{p}\left(\omega_{0}\right) & =\left.\omega_{0} \frac{d \angle G_{\tau}(j \omega)}{d \omega}\right|_{\omega_{0}}=\left.\omega_{0} \frac{d \angle G(j \omega)}{d \omega}\right|_{\omega_{0}}-\tau \omega_{0} \approx \angle G\left(j \omega_{0}\right)+\frac{2}{\pi}\left[\ln \left|K_{g}\right|-\ln \left|G\left(j \omega_{0}\right)\right|\right]-\tau \omega_{0} \\
& \approx \angle G_{\tau}\left(j \omega_{0}\right)+\frac{2}{\pi}\left[\ln \left|K_{g}\right|-\ln \left|G_{\tau}\left(j \omega_{0}\right)\right|\right]
\end{aligned}
$$

The above relations show that the pure time delay should be known for the calculation of $s_{a}\left(\omega_{0}\right)$ but it has no effect on the calculation of $s_{p}\left(\omega_{0}\right)$. It should be remembered that $\tau$ represents the pure time delay of the system which is usually related to the mass transport delay and is often negligible or can be easily measured. This value should not be confounded with the time delay that is used to model a high-order system as a first- or second-order system with delay. For example, $G(s)=1 /(s+1)^{5}$ has no pure time delay whereas it can be approximated by a first-order model with a large time delay.

\subsection{PID design}

Suppose that the amplitude and the phase of a plant at the crossover frequency $\omega_{c}$ are known. These values may be obtained using the existing controller and by the method mentioned in Section 2. Suppose also that the static gain of the process is measured. The objective is to improve the controller performance by adjusting the phase margin and the slope of the Nyquist curve at the crossover frequency. The modified Ziegler-Nichols method is used but the derivatives are approximated by the Bode's integrals, so no model for the system is required. To obtain a desired phase margin $\Phi_{d}$ at the crossover frequency $\omega_{c}$ we have the following equations to solve:

$$
\begin{aligned}
& \angle G\left(j \omega_{c}\right)+\angle K\left(j \omega_{c}\right)=\Phi_{d}-\pi \\
& \left|G\left(j \omega_{c}\right) K\left(j \omega_{c}\right)\right|=1
\end{aligned}
$$


Solving these equations one obtains:

$$
\begin{gathered}
K_{p}=\frac{\cos \left(\Phi_{d}-\varphi_{c}-\pi\right)}{\left|G\left(j \omega_{c}\right)\right|} \\
T_{d} \omega_{c}-\frac{1}{T_{i} \omega_{c}}=\tan \left(\Phi_{d}-\varphi_{c}\right)
\end{gathered}
$$

where $\varphi_{c}$ is the phase of $G\left(j \omega_{c}\right)$. Now we exploit Eq. (10) in order to obtain the desired slope $\psi$ at the crossover frequency. Combining Eqs (10) and (26), we obtain after straightforward calculations the parameters $T_{i}$ and $T_{d}$ as follows:

$$
\begin{aligned}
T_{i} & =\frac{1}{\omega_{c}\left(T_{d} \omega_{c}-\tan \left(\Phi_{d}-\varphi_{c}\right)\right)} \\
T_{d} & =\frac{1}{2 \omega_{c}}\left[\left(s_{a}\left(\omega_{c}\right)-s_{p}\left(\omega_{c}\right) \tan \left(\Phi_{d}-\varphi_{c}\right)\right) \tan \left(\psi-\varphi_{c}\right)+\left(1-s_{a}\left(\omega_{c}\right)\right) \tan \left(\Phi_{d}-\varphi_{c}\right)-s_{p}\left(\omega_{c}\right)\right]
\end{aligned}
$$

The improved PID controller is now defined by (25), (27) and (28).

\subsection{Example}

The PID design method presented above will be illustrated by the following simulation model:

$$
G(s)=\frac{1}{(s+1)^{5}}
$$

The specifications are set at $0.4 \mathrm{rad} / \mathrm{s}$ for the crossover frequency and $50^{\circ}$ for the phase margin. First, the control parameters are obtained using the modified Ziegler-Nichols method. The resulting PID controller is

$$
K(s)=1.35\left(1+\frac{1}{3.44 s}+0.86 s\right)
$$

This controller moves the point $G(0.4 j)$ of the Nyquist curve to a point of $K(j \omega) G(j \omega)$ on the unit circle having a phase of $130^{\circ}$. This conforms to the closed-loop system with the specifications mentioned above.

In order to improve the closed-loop performance, let us calculate now a controller that provides the closed-loop system the same crossover frequency and phase margin, but with the desired slope of the openloop Nyquist curve at the crossover frequency of $65^{\circ}$. This reduces the current slope of the Nyquist curve by $25^{\circ}$ and ensures a greater distance of the Nyquist curve from the critical point in high frequencies. The controller parameters are obtained from Eqs $(25),(28)$ and $(27)$, where $s_{a}(0.4 j)$ and $s_{p}(0.4 j)$ are approximated using Eqs (13) and (17):

$$
K(s)=1.35\left(1+\frac{1}{2.81 s}+1.27 s\right)
$$

Although the approximation error for $s_{a}$ and $s_{p}$ leads to a resultant slope of $74^{\circ}$ (about $13 \%$ error), a comparison of the closed-loop performance for the two controllers shown in Fig. 5 illustrates a significant improvement of the closed-loop performance. The overshoot is about the same but the settling time is $44 \%$ smaller with the proposed method. it should be mentioned that, in the simulation, the derivative 
part of the ideal PID controller is replaced by a causal derivator $\frac{T_{d} s}{1+T_{d} / 20 s}$ and no saturation is considered for the control signal. The Nyquist diagrams (Fig. 6) further show that the proposed controller modifies the slope of the Nyquist curve and as a result improves the gain margin as well as the modulus margin (the minimum distance between $L(j \omega)$ and the critical point) of the system.
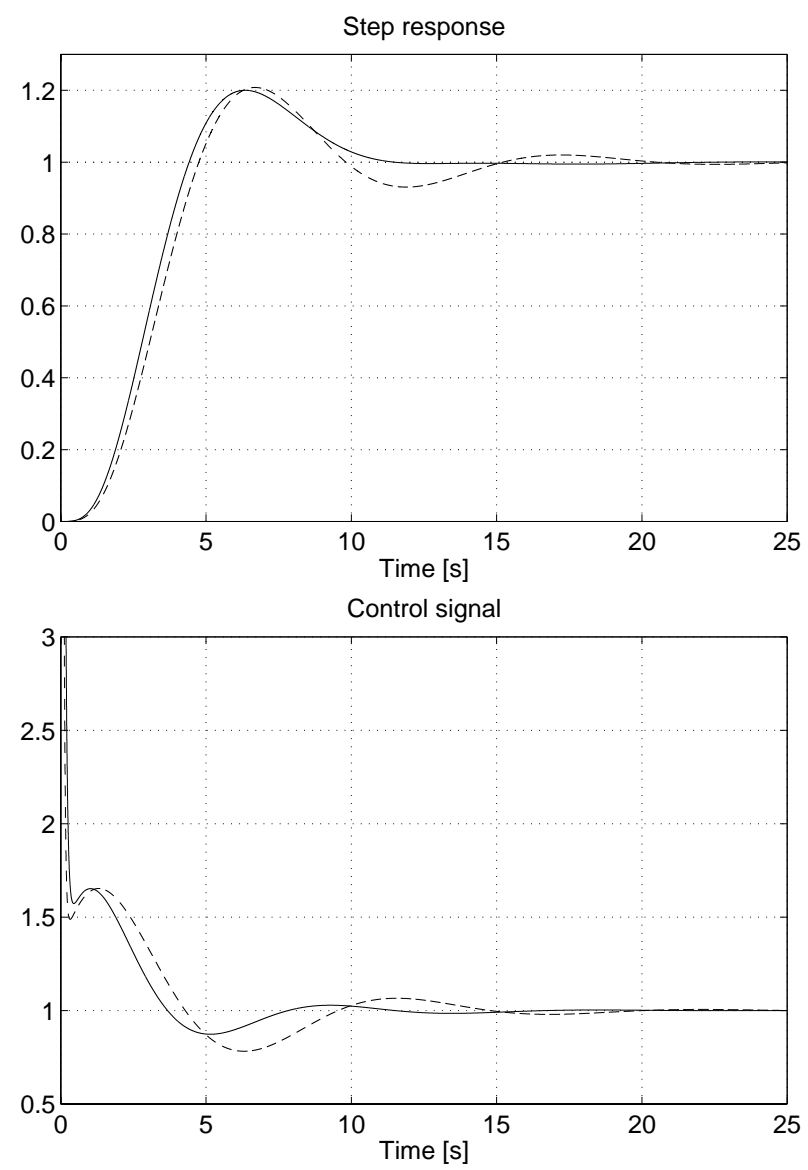

Figure 5: Step response of the closed-loop system (dashed line: modified Ziegler-Nichols, solid line: proposed)

\section{Iterative PID tuning in closed-loop}

In the previous section, it was assumed that the amplitude and the phase of the plant at the crossover frequency are known or they are measured by a relay feedback experiment with an existing controller. However, if the desired crossover frequency is different from the measured one, an analytic solution as presented in the previous section cannot be obtained. Therefore it is not possible, for example, to improve the performance of the closed-loop system by increasing the crossover frequency which is closely related to closed-loop bandwidth. In addition, although the Nyquist curve of the loop transfer function passes 


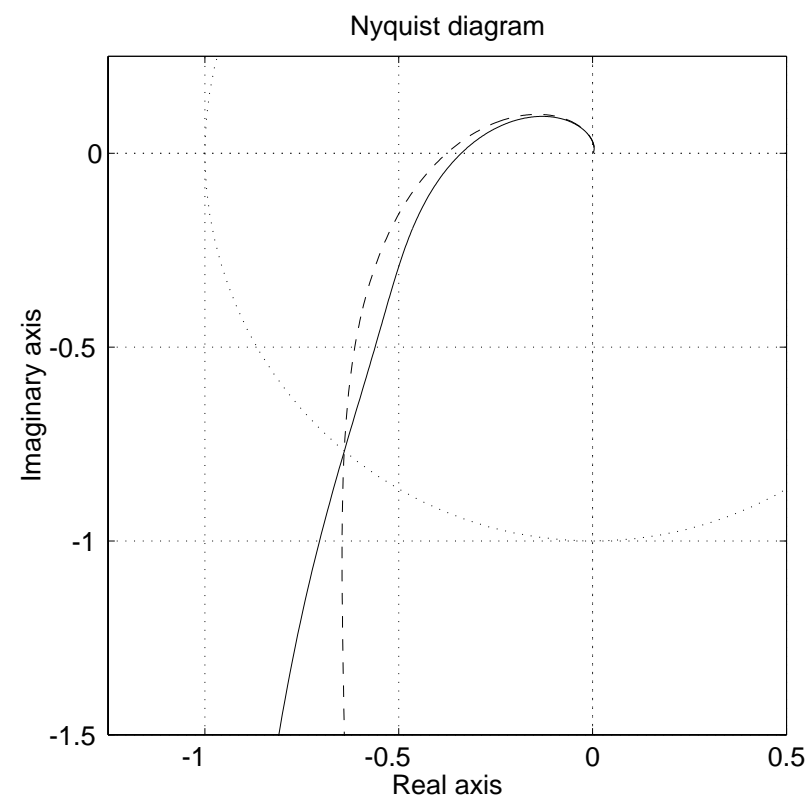

Figure 6: Nyquist diagram (dashed line: modified Ziegler-Nichols, solid line: proposed)

through a point with the desired phase, it is not guaranteed that this point gives the phase margin (it is possible that the Nyquist curve intersects several times the unit circle). So an extra relay feedback test using the new controller should be carried out in order to validate the desired performance on the real system.

In this section an iterative tuning method in the frequency domain is proposed. The method is based on the measurement of the phase margin and the crossover frequency by the closed-loop relay test of Section 2 improved by an adaptive gain adjustment proposed in [14]. In this method, a frequency criterion is minimized iteratively using the Gauss-Newton algorithm. The particularity of this method is that the gradient of the criterion is approximated using the Bode's integrals and no model of the plant is required.

\subsection{Iterative procedure for phase margin adjustment}

First of all, a performance criterion in the frequency domain is defined as follows:

$$
J(\rho)=\frac{1}{2}\left[\left(\frac{\omega_{c}-\omega_{d}}{\omega_{d}}\right)^{2}+\left(\frac{\Phi_{m}-\Phi_{d}}{\Phi_{d}}\right)^{2}\right]
$$

where $\rho$ is the vector of the controller parameters, $\omega_{c}$ and $\omega_{d}$ are respectively the measured and desired crossover frequencies, and $\Phi_{m}$ and $\Phi_{d}$ are the measured and desired phase margins. The error between the measured and the desired values are normalized in order that the same weighting for each variable in the criterion to be obtained. This avoids the numerical problems in the iterative algorithm. In the next step, the controller parameters minimizing the criterion are obtained by the iterative Newton formula:

$$
\rho_{i+1}=\rho_{i}-\gamma_{i} R^{-1} J^{\prime}\left(\rho_{i}\right)
$$


where $i$ is the iteration number, $\gamma_{i}$ is a positive scalar representing the step size, $R$ is a square positive definite matrix of dimension $\rho$ and $J^{\prime}(\rho)$ is the gradient of the criterion with respect to $\rho$. This algorithm converges to the vector of parameters minimizing the criterion, provided that the step size is properly chosen [5]. The matrix $R$ can be chosen equal to the identity matrix or to the Hessian of the criterion to obtain faster convergence (Gauss-Newton method).

It should be mentioned that the slope of the loop Nyquist curve can also be adjusted simultaneously in the iterative approach. In fact, in this case, the vector of parameters contains only $K_{p}$ and $T_{i}$ and Eq. (10) is used at each iteration to compute $T_{d}$.

The gradient of the criterion is given by:

$$
J^{\prime}(\rho)=\frac{1}{\omega_{d}^{2}}\left(\omega_{c}-\omega_{d}\right) \frac{\partial \omega_{c}}{\partial \rho}+\frac{1}{\Phi_{d}^{2}}\left(\Phi_{m}-\Phi_{d}\right) \Phi_{m}^{\prime}
$$

where $\Phi_{m}^{\prime}$ is the derivative of the phase margin with respect to $\rho$ computed through the chain rule (note that $\Phi_{m}$ is a function of $\rho$ and $\omega_{c}$ ):

$$
\Phi_{m}^{\prime}=\frac{\partial \Phi_{m}}{\partial \rho}+\left.\frac{\partial \Phi_{m}}{\partial \omega}\right|_{\omega_{c}} \frac{\partial \omega_{c}}{\partial \rho}
$$

Now replacing $\Phi_{m}$ in the above equation by $\angle L\left(j \omega_{c}\right)+\pi$ gives:

$$
\Phi_{m}^{\prime}=\frac{\partial L L\left(j \omega_{c}\right)}{\partial \rho}+\left.\frac{\partial L L(j \omega)}{\partial \omega}\right|_{\omega_{c}} \frac{\partial \omega_{c}}{\partial \rho}
$$

The first term in the above equation is equal to $\partial \angle K\left(j \omega_{c}\right) / \partial \rho$ which is completely known at each iteration. Furthermore one has:

$$
\left.\frac{\partial \angle L(j \omega)}{\partial \omega}\right|_{\omega_{c}}=\left.\frac{\partial \angle K(j \omega)}{\partial \omega}\right|_{\omega_{c}}+\left.\frac{\partial \angle G(j \omega)}{\partial \omega}\right|_{\omega_{c}}=\left.\frac{\partial \angle K(j \omega)}{\partial \omega}\right|_{\omega_{c}}+\frac{s_{p}\left(\omega_{c}\right)}{\omega_{c}}
$$

Again, the first term is completely known and $s_{p}\left(\omega_{c}\right)$ can be approximated using Eq. (17). Now, it only remains to compute $\partial \omega_{c} / \partial \rho$. For this purpose, we use the fact that the loop gain at $\omega_{c}$ in each iteration is equal to 1 , therefore its derivative (or derivative of its logarithm) with respect to $\rho$ will be zero. Then one has:

$$
\frac{\partial \ln \left|L\left(j \omega_{c}\right)\right|}{\partial \rho}+\left.\frac{\partial \ln |L(j \omega)|}{\partial \omega}\right|_{\omega_{c}} \frac{\partial \omega_{c}}{\partial \rho}=0
$$

but from the Bode's integral (see Eq. (13)) one has:

$$
\left.\frac{\partial \ln |L(j \omega)|}{\partial \omega}\right|_{\omega_{c}} \approx \frac{2 \angle L\left(j \omega_{c}\right)}{\pi \omega_{c}}=\frac{2\left(\Phi_{m}-\pi\right)}{\pi \omega_{c}}
$$

Thus $\partial \omega_{c} / \partial \rho$ can be approximated as follows (note that $\partial \ln |L(j \omega)| / \partial \rho=\partial \ln |K(j \omega)| / \partial \rho$ ):

$$
\frac{\partial \omega_{c}}{\partial \rho} \approx-\frac{\pi \omega_{c}}{2\left(\Phi_{m}-\pi\right)} \frac{\partial \ln \left|K\left(j \omega_{c}\right)\right|}{\partial \rho}
$$

Notice that the gradient of the criterion is computed using only the measured crossover frequency $\omega_{c}$ and the measured amplitude and phase of the plant. In the same way, the Hessian of the criterion can be 
approximated without any additional information as follows:

$$
H=J^{\prime \prime}(\rho)=\frac{1}{\omega_{d}^{2}} \frac{\partial \omega_{c}}{\partial \rho}\left(\frac{\partial \omega_{c}}{\partial \rho}\right)^{T}+\frac{1}{\Phi_{d}^{2}} \Phi_{m}^{\prime}\left(\Phi_{m}^{\prime}\right)^{T}+\frac{1}{\omega_{d}^{2}}\left(\omega_{c}-\omega_{d}\right) \frac{\partial^{2} \omega_{c}}{\partial \rho^{2}}+\frac{1}{\Phi_{d}^{2}}\left(\Phi_{m}-\Phi_{d}\right) \Phi_{m}^{\prime \prime}
$$

The last two terms can be neglected because they are small especially in the neighborhood of the final solution. In addition, this simplification makes the Hessian always positive which fixes the numerical problems normally encountered in the iterative Newton algorithm. The use of the Hessian matrix in the iterative formula (Eq. 33) instead of $R$ significantly improves the convergence speed.

$$
R=H \approx \frac{1}{\omega_{d}^{2}} \frac{\partial \omega_{c}}{\partial \rho}\left(\frac{\partial \omega_{c}}{\partial \rho}\right)^{T}+\frac{1}{\Phi_{d}^{2}} \Phi_{m}^{\prime}\left(\Phi_{m}^{\prime}\right)^{T}
$$

\subsection{Iterative procedure for phase and gain margins adjustment}

In phase margin and slope adjustment, the controller is designed using only the information on one frequency point of the plant. Although this approach is very fast and simple and works well for the majority of the industrial processes, it may not work appropriately for some complex high-order systems. This means that the specifications may be satisfied just locally at the crossover frequency and it is possible that the behavior of the system changes drastically elsewhere, leading for example to a very small gain margin. For such systems, it may be preferable to tune the phase and gain margins at the same time. However, this necessitates two relay experiments in each iteration: one relay test to measure the gain margin and the other for the phase margin. A frequency criterion can be defined as follows:

$$
J(\rho)=\frac{1}{2}\left[\left(\frac{\omega_{c}-\omega_{d}}{\omega_{d}}\right)^{2}+\left(\frac{\Phi_{m}-\Phi_{d}}{\Phi_{d}}\right)^{2}+\left(\frac{K_{u}-K_{d}}{K_{d}}\right)^{2}\right]
$$

where $K_{u}=\left|L\left(j \omega_{u}\right)\right|$ is the amplitude of the loop transfer function where it intersects the negative real axis $\left(\angle L\left(j \omega_{u}\right)=-\pi\right) . \omega_{u}$ is the critical loop frequency, $K_{d}$ is the inverse of the desired gain margin. The gradient of the criterion is given by:

$$
J^{\prime}(\rho)=\frac{1}{\omega_{d}^{2}}\left(\omega_{c}-\omega_{d}\right) \frac{\partial \omega_{c}}{\partial \rho}+\frac{1}{\Phi_{d}^{2}}\left(\Phi_{m}-\Phi_{d}\right) \Phi_{m}^{\prime}+\frac{1}{K_{d}^{2}}\left(K_{u}-K_{d}\right) K_{u}^{\prime}
$$

where $K_{u}^{\prime}$ is the derivative of the critical loop gain with respect to $\rho$ computed as follows:

$$
K_{u}^{\prime}=\frac{\partial\left|L\left(j \omega_{u}\right)\right|}{\partial \rho}+\left.\frac{\partial|L(j \omega)|}{\partial \omega}\right|_{\omega_{u}} \frac{\partial \omega_{u}}{\partial \rho}
$$

The first term is equal to $\left|G\left(j \omega_{u}\right)\right| \cdot \partial\left|K\left(j \omega_{u}\right)\right| / \partial \rho$ which can be easily computed at each iteration. The second term can be approximated using the Bode's integrals in a similar way as is done for the phase margin. First the derivative of the amplitude of the loop gain is computed using Eq. (13) as follows:

$$
\left.\frac{\partial|L(j \omega)|}{\partial \omega}\right|_{\omega_{u}}=\left.\left|L\left(j \omega_{u}\right)\right| \frac{\partial \ln |L(j \omega)|}{\partial \omega}\right|_{\omega_{u}} \approx K_{u} \frac{2 L L\left(j \omega_{u}\right)}{\pi \omega_{u}}=-\frac{2 K_{u}}{\omega_{u}}
$$

Then $\partial \omega_{u} / \partial \rho$ is computed using the fact that in each iteration $\angle L\left(j \omega_{u}\right)=-\pi$ and consequently its derivative with respect to $\rho$ will be zero, which gives:

$$
\frac{\partial L L\left(j \omega_{u}\right)}{\partial \rho}+\left.\frac{\partial L L(j \omega)}{\partial \omega}\right|_{\omega_{u}} \frac{\partial \omega_{u}}{\partial \rho}=0
$$


The first term in the above equation is equal to $\partial \angle K\left(j \omega_{u}\right) / \partial \rho$ which can be computed knowing the controller at each iteration. Furthermore one has:

$$
\left.\frac{\partial \angle L(j \omega)}{\partial \omega}\right|_{\omega_{u}}=\left.\frac{\partial \angle K(j \omega)}{\partial \omega}\right|_{\omega_{u}}+\left.\frac{\partial \angle G(j \omega)}{\partial \omega}\right|_{\omega_{u}}
$$

Again, knowing the controller at each iteration, the first term in the right hand side can be computed while the second term is approximated using the Bode's integral of Eq. (17). Therefore $\partial \omega_{u} / \partial \rho$ is given by:

$$
\frac{\partial \omega_{u}}{\partial \rho}=-\left(\left.\frac{\partial \angle K(j \omega)}{\partial \omega}\right|_{\omega_{u}}+\frac{s_{p}\left(\omega_{u}\right)}{\omega_{u}}\right)^{-1} \frac{\partial \angle K\left(j \omega_{u}\right)}{\partial \rho}
$$

In a similar way an approximation of the Hessian can also be computed as follows:

$$
R=H \approx \frac{1}{\omega_{d}^{2}} \frac{\partial \omega_{c}}{\partial \rho}\left(\frac{\partial \omega_{c}}{\partial \rho}\right)^{T}+\frac{1}{\Phi_{d}^{2}} \Phi_{m}^{\prime}\left(\Phi_{m}^{\prime}\right)^{T}+\frac{1}{K_{d}^{2}} K_{u}^{\prime}\left(K_{u}^{\prime}\right)^{T}
$$

\subsection{Simulation example}

Now let us consider a complex plant model including a time delay as follows:

$$
G(s)=\frac{e^{-0.3 s}}{\left(s^{2}+2 s+3\right)^{3}(s+3)}
$$

This model was proposed in [13] and represents a difficult problem to be solved using a PID controller. It should be noted that neither the traditional Ziegler-Nichols method nor a more advanced method based on a first-order plant model [6] can give a stabilizing controller for this plant. Contrary to the simulation example presented in Section 3.4, it is very difficult here to tune a controller using the slope adjustment method, because a little change in the slope of the Nyquist curve at the crossover frequency may even make the closed-loop system unstable. In the following, it is shown that a controller which confers appropriate specifications on the closed-loop system can be tuned with the iterative phase and gain margins adjustment method. This method requires two experiments per iteration and is more convenient for high-order complex systems. However, one should note that the experimental time for the gain margin measurement can be chosen to be smaller than that of the phase margin, because the critical frequency is appreciably larger than the crossover frequency.

The specifications are set for this example at $\omega_{d}=0.2 \mathrm{rad} / \mathrm{s}$ for the crossover frequency, and $\Phi_{d}=70^{\circ}$ and $K_{d}=\frac{1}{3}$ for the phase and for the inverse of the gain margin, respectively. The initial controller is designed using the Kappa-Tau tuning rule proposed in [8] which gives the following stabilizing controller:

$$
K(s)=4.5\left(1+\frac{1}{0.41 s}+0.033 s\right)
$$

The performance criterion of Eq. (43) is considered. Two closed-loop experiments are performed to measure a crossover frequency of $0.139 \mathrm{rad} / \mathrm{s}$, a phase margin of $78.5^{\circ}$ and a gain margin of 4.39 , which 
give a performance criterion of 0.104 . With these values, the gradient and Hessian are calculated, and a new controller is obtained. After one other iteration the following controller is obtained:

$$
K(s)=4.93\left(1+\frac{1}{0.316 s}+0.125 s\right)
$$

with a performance criterion of 0.0017 . This controller gives $0.1997 \mathrm{rad} / \mathrm{s}$ for the crossover frequency, $66.0^{\circ}$ for the phase margin and 2.97 for the gain margin. It should be noted that no significant improvement can be achieved with further iterations. A comparison of the closed-loop performance between the initial controller and the final one in Fig. 7 shows that a much smaller settling time is achieved with an overshoot of only $0.7 \%$. In the simulation example, $T_{d} s$ is replaced by a causal derivator $\frac{T_{d} s}{1+T_{d} / 20 s}$.
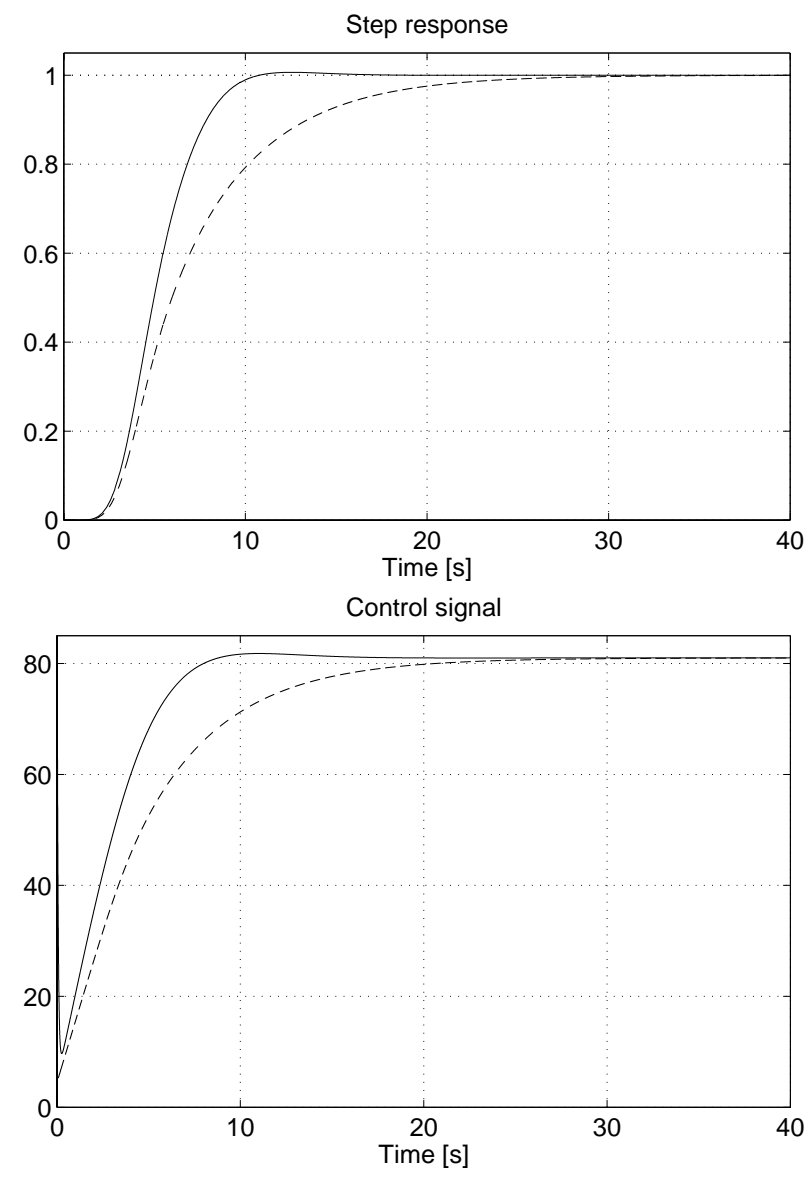

Figure 7: Step response of the closed-loop system (dashed line: Kappa-Tau, solid line: proposed)

It is worth mentioning that even if the estimation errors of $s_{a}$ might be large due to the presence of an unknown pure time delay in the plant model, the criterion converges rapidly enough. The reason is that in the Gauss-Newton algorithm the quadratic criterion will converge to its minimum value even with an approximative gradient. 


\section{Real-time experiment}

In this section, experimental results obtained with a three-tank system (Fig. 8) are presented. The process consists of three cylinders T1, T2 and T3 which are interconnected in series by two connecting pipes. Two pumps, driven by DC motors, supply the tanks T1 and T2 with the liquid (water) collected in a reservoir. The water level in each tank is measured by a piezo-resistive pressure transducer. The first tank is equipped with a manually adjustable valve which lets the water outflow at rate $Q_{\text {out }}$ to the reservoir. The input $u$ of the process is an electrical signal which controls the flow rate $Q_{1}$ into the first tank. The output $y$ of the process is the voltage of the piezo-resistive pressure transducer of $\mathrm{T} 2$, which is proportional to the level $L$ with a negative gain. The second pump acts as an output disturbance to the system. The control objective is to maintain the level of T2 at a desired value. Due to nonlinear relations between the input flow rate $Q_{1}$, the output flow rate $Q_{\text {out }}$ and the tanks' levels, the transfer function of the considered system is nonlinear.

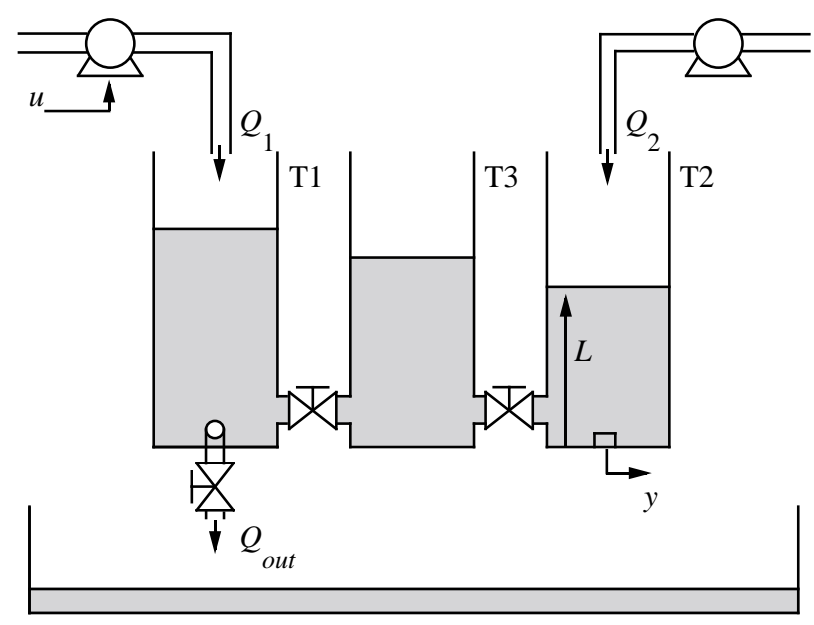

Figure 8: Schematic diagram of the three-tank system

Consider that the system operates at a set point corresponding to $Q_{1}=\frac{Q_{1 \max }}{2}$, where $Q_{1 \max }$ is the maximum flow rate of the pump. For this operating point, an initial controller is designed using the KappaTau tuning rule proposed in [8] based on the step response of the system. The resulting PID controller is:

$$
K_{\kappa \tau}(s)=29.3\left(1+\frac{1}{20.84 s}+4.72 s\right)
$$

It should be noted that the Ziegler-Nichols method gives an unstable controller for this operating point. After measuring a static gain of about 1, a closed-loop experiment as proposed in Section 2 identifies for this configuration a phase margin of $64^{\circ}$ and a crossover frequency of $0.097 \mathrm{rad} / \mathrm{s}$. In order to improve the stability of the closed-loop system and reduce the control effort, new specifications with an increased phase margin $\Phi_{d}$ and a reduced crossover frequency $\omega_{d}$ compared with the actual ones, are defined: $\Phi_{d}=80^{\circ}$, 
$\omega_{d}=0.08 \mathrm{rad} / \mathrm{s}$. As another specification, a desired value for the slope of the Nyquist curve at crossover frequency or a constant ratio between the integral time and the derivative time should be considered. In this experiment, like in the classical Ziegler-Nichols, $T_{i}=4 T_{d}$ is considered and the derivative part of the controller is replaced by a causal derivator $\frac{T_{d} s}{1+T_{d} / 20 s}$. In the next step the gradient and the Hessian are calculated and a new controller is obtained after the first iteration:

$$
K(s)=20.4\left(1+\frac{1}{31.5 s}+7.88 s\right)
$$

The closed-loop test applied to the system with the controller in Eq.(55) gives the following estimates:

$$
\Phi_{m}=86.2^{\circ} \quad \omega_{m}=0.085 \mathrm{rad} / \mathrm{s}
$$

As the estimated values are close to the desired ones, there is no need for further iterations. A comparison between the time response of the closed-loop system with the Kappa-Tau controller and the proposed one is shown in Fig. 9. The step response is normalized and the output disturbance concerns a constant flow rate $Q_{2}$ for the pump 2 applied at $t=160 \mathrm{~s}$. It can be seen that the proposed controller gives a much better performance for the closed-loop system in terms of disturbance rejection, overshoot and settling time.

Now, consider that the operating point of the system is changed and, due to the system nonlinearity, the closed-loop performance deteriorates. Consequently, the same controller at the new operating point does not meet the specification (a phase margin $\Phi_{m}$ of $=55^{\circ}$ and a crossover frequency $\omega_{m}$ of $0.066 \mathrm{rad} / \mathrm{s}$ are obtained) and the controller should be retuned. After one iteration, using the proposed tuning method, the following controller is obtained:

$$
K(s)=23\left(1+\frac{1}{42.3 s}+10.6 s\right)
$$

The closed-loop system with this controller gives the following performance which is very close to the desired one:

$$
\Phi_{m}=73.7^{\circ} \quad \omega_{m}=0.0791 \mathrm{rad} / \mathrm{s}
$$

A comparison of the normalized step response and disturbance response of the closed-loop system with the two controllers is shown in Fig. 10. The effectiveness and rapidity of the proposed algorithm is shown in Fig. 11 for an autotuning experiment. During the first 500 seconds the initial controller is implemented on the real system and measurements are performed. Then the controller is updated and a new test is carried out for measuring the phase margin as well as the crossover frequency.

As shown in this section, the proposed iterative tuning algorithm is fast enough to be used for the autotuning of real processes, and is particularly appropriate for readjusting the controller parameters of systems whose operating point changes slowly. 

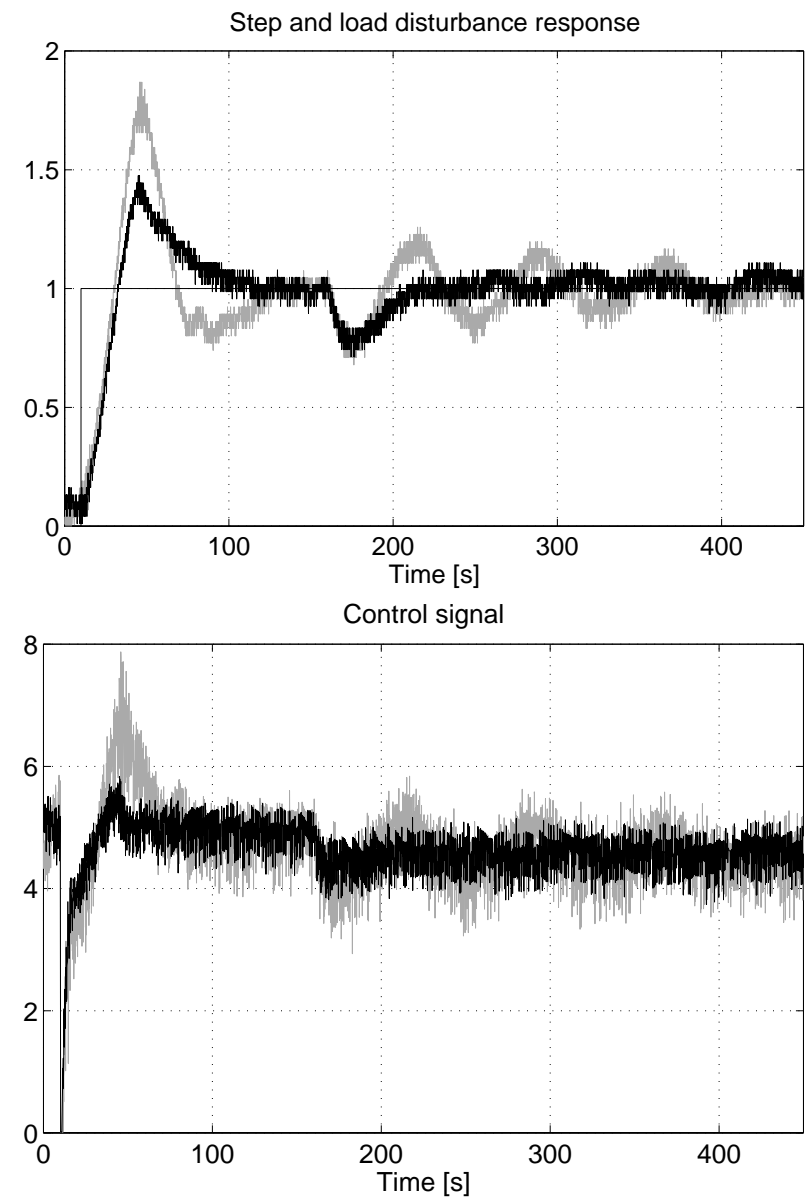

Figure 9: Normalized step and disturbance response of the closed-loop system (grey line: Kappa-Tau, black line: proposed)

\section{Conclusions}

The derivatives of phase and amplitude of minimum-phase and stable plant models with respect to the frequency have been approximated using the Bode's integrals. The precision of the approximation for typical industrial plant models is adequate for PID controller tuning. An iterative approach for tuning the PID controller parameters with specifications on gain margin, phase margin and crossover frequency was proposed. This approach takes advantage of Bode's integrals to estimate the gradient and the Hessian of a frequency criterion and therefore requires no parametric model of the plant. The proposed iterative approach converges in a few iterations to the minimum of the frequency criterion and can be used for autotuning of industrial plants. 

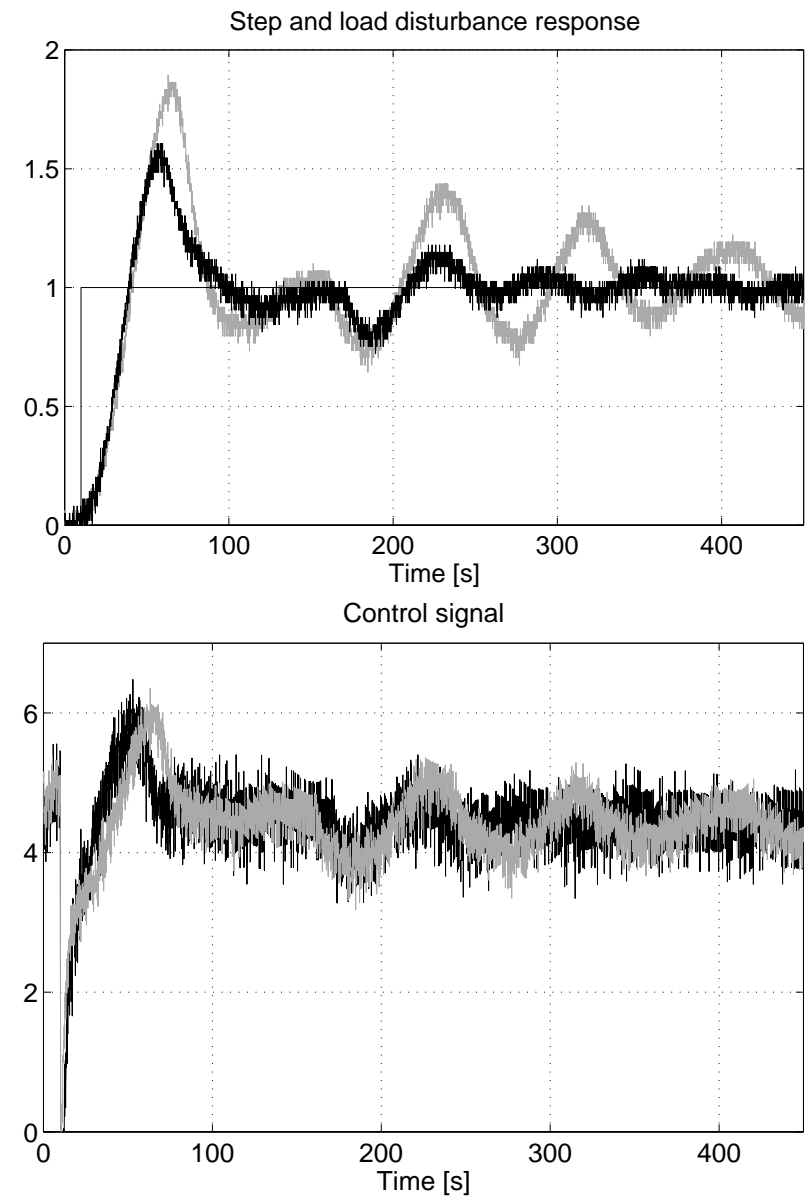

Figure 10: Normalized step and disturbance response of the closed-loop system (grey line: controller (55), black line: controller (56))

\section{References}

[1] A. Besancon-Voda and H. Roux-Buisson. Another version of the relay feedback experiment. Journal of Process Control, 7(4):303-308, 1997.

[2] H. W. Bode. Network Analysis and Feedback Amplifier Design. New York, Van Nostrand, 1945.

[3] A. Datta, M. T. Ho, and S. P. Bhattacharyya. Structure and synthesis of PID Controllers. SpringerVerlag London, 2000.

[4] G. H. M. de Arruda and P. R. Barros. Relay based gain and phase margins PI controller design. In IEEE Instrumentation and Measurement Technology Conference, Budapest, May 21-23, pages 11891194, 2001.

[5] J. E. Dennis and R. B. Schnabel. Numerical Methods for Unconstrained Optimization and Nonlinear Equations. SIAM, 1996. 


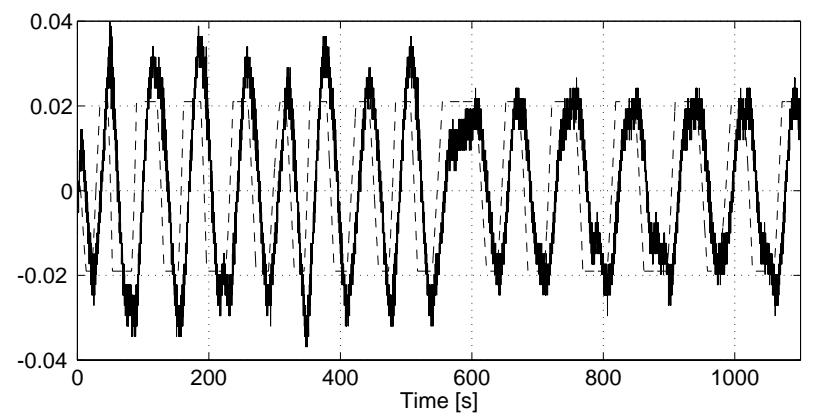

Figure 11: Autotuning experiment (dashed: generated reference signal of the closed-loop system, solid: output of the closed-loop system)

[6] W. K. Ho, C. C. Hang, and L. S. Cao. Tuning of PID controllers based on gain and phase margin specifications. Automatica, 31(3):497-502, 1995.

[7] K. J. Aström and T. Hägglund. Automatic tuning of simple regulators with specifications on phase and amplitude margins. Automatica, 20(5):645-651, 1984.

[8] K. J. Aström and T. Hägglund. PID Controllers: Theory, Design and Tuning. Instrument Society of America, 2nd edition, 1995.

[9] B. Kristiansson, B. Lennartson, and C. M. Fransson. From PI to $H_{\infty}$ control in a unified framework. In 39th IEEE-CDC, Sydney, Australia, pages 2740-2745, 2000.

[10] A. Leva. PID autotuning algorithm based on relay feedback. IEE Proceedings-D, 140(5):328-338, September 1993.

[11] R. Longchamp and Y. Piguet. Closed-loop estimation of robustness margins by the relay method. IEEE ACC, pages 2687-2691, 1995.

[12] Q. G. Wang, H. W. Fung, and Y. Zhang. PID tuning with exact gain and phase margins. ISA Transactions, (38):243-249, 1999.

[13] Q. G. Wang, T. H. Lee, H. W. Fung, Q. Bi, and Y. Zhang. PID tuning for improved performance. IEEE Transactions on CST, 7(4):3984-3989, 1999.

[14] M. Saeki. A new adaptive identification method of critical loop gain for multi-input multi-output plants. 37th IEEE CDC, 4:3984-3989, 1998.

[15] T. S. Schei. Closed-loop tuning of PID controllers. In ACC, FA12, pages 2971-2975, 1992.

[16] C. C. Yu. Autotuning of PID Controllers: Relay Feedback Approach. Springer-Verlag London, 1999. 